\title{
Optimization of a simple vitrification procedure for bovine embryos produced in vitro: effect of developmental stage, two-step addition of cryoprotectant and sucrose dilution on embryonic survival
}

\author{
A. R. Mahmoudzadeh ${ }^{1 *}$, A. Van Soom ${ }^{1}$, P. Bols ${ }^{1}$, M. T. Ysebaert ${ }^{2}$ and \\ A. de Kruif ${ }^{1}$ \\ ${ }^{1}$ Department of Reproduction, Obstetrics and Herd Health and ${ }^{2}$ Department of Physiology, Biochemistry \\ and Biometrics, Faculty of Veterinary Medicine, State University of Ghent, Casinoplein 24, 9000 Ghent, \\ Belgium
}

\begin{abstract}
Experiments were designed to determine optimal conditions for the cryopreservation of bovine embryos produced in vitro. In Expt 1, embryos were exposed for 1, 3 or 5 min to a vitrification solution consisting of $40 \%(\mathrm{v} / \mathrm{v})$ ethylene glycol, $18 \%(\mathrm{w} / \mathrm{v})$ Ficoll and $10.26 \%$ $(\mathrm{w} / \mathrm{v})$ sucrose (EFS) and were subsequently vitrified. After warming in water at room temperature and diluting in a solution of $0.25 \mathrm{~mol}_{\text {sucrose }} \mathrm{l}^{-1}$, the in vitro survival rate in Ménézo-B2 medium was highest after exposure to EFS for 1 min. In Expt 2, embryos at day 7 and day 8 were vitrified after exposure to EFS for $I \mathrm{~min}$. The survival rate of embryos at day 7 was significantly improved, especially at the blastocyst and expanded blastocyst stage, when the Ménézo-B2 medium was supplemented with bovine oviduct epithelial cells (BOEC). Embryos at day 8 exhibited a significantly lower survival rate than did embryos at day 7 in both culture media. In Expt 3, one-step exposure of embryos to EFS for 1 min was compared with two-step exposure to $20 \%$ ethylene glycol for $3 \mathrm{~min}$ and EFS for $30-45 \mathrm{~s}$. Embryos exhibited significantly higher survival and hatching rates after two-step vitrification, especially at the expanded blastocyst ( $89 \%$ and $69 \%$, respectively) and the blastocyst stage ( $75 \%$ and $38 \%$, respectively). In Expt 4 , embryos were diluted in solutions of $0,0.25$ or $0.5 \mathrm{~mol}$ sucrose $\mathrm{I}^{-1}$ after two-step vitrification. There were no significant differences in the survival rates between the three dilution treatments. It can be concluded that (i) the optimal exposure time to EFS for one-step vitrification is $1 \mathrm{~min}$; (ii) embryonic survival depends on the developmental stage; (iii) the addition of BOEC to culture medium after warming is beneficial for culture of vitrified embryos in vitro; (iv) two-step addition of EFS improves the survival rate and (v) vitrified embryos can be diluted from EFS in a single step without the use of sucrose as an osmotic buffer.
\end{abstract}

\section{Introduction}

Cryopreservation of cattle embryos is indispensable for economic and scientific reasons. Currently, there are two basic approaches towards cryopreservation of embryos. The first approach consists of a controlled, slow freezing process that was first developed for mouse embryos (Whittingham, 1971), and later applied to bovine embryos produced in vivo (Wilmut and Rowson, 1973). The second approach, vitrification, involves the addition of higher concentrations of cryoprotectant and a very rapid freezing rate, which prevents the formation of ice crystals (Fahy et al., 1984). The first successful vitrification of flushed bovine morulae and early blastocysts was reported by Massip et al. (1986).

\footnotetext{
*Present address: Department of Animal Science, Faculty of Agriculture, Gilan University, PO Box 3179, Rasht, Iran.

Received 2 March 1994.
}

Methods that were initially developed for conventional cryopreservation of bovine embryos produced in vivo were subsequently applied to cattle embryos produced by in vitro maturation and fertilization techniques (Massip et al., 1993; Zhang et al., 1993; Shamsuddin et al., 1994). However, recent data indicate that rapid cooling, rather than slow cooling, may be beneficial for the survival of cattle embryos produced in vitro (Leibo and Loskutoff, 1993; Mahmoudzadeh et al., 1994b; Pollard and Leibo, 1994). Vitrification of cattle embryos produced in vitro has been very successful with the use of various cryoprotectants, including a mixture of glycerol and 1,2-propanediol (Kuwayama et al., 1992) and a vitrification solution, EFS, consisting of ethylene glycol, Ficoll and sucrose (Tachikawa et al., 1993). In the latter study, a $74-77 \%$ survival rate of bovine blastocysts was obtained after warming and culturing the vitrified embryos in vitro. EFS, which was first described by Kasai et al. (1990) for mouse embryos at the morula stage, has also been used successfully for the 
vitrification of rabbit embryos (Kasai et al., 1992), bovine morulae produced in vivo (Mahmoudzadeh et al., 1993), bovine blastocysts produced in vitro (Tachikawa et al., 1993) and mouse expanded blastocysts (Zhu et al., 1993). On the basis of these reports, different experiments were designed to optimize the cryopreservability of cattle embryos produced in vitro. The effect of time of exposure to EFS, the influence of embryonic developmental stage, the effect of the addition of bovine oviduct epithelial cells to the culture medium after warming, the effect of one- and two-step addition of EFS, and the need for sucrose in the dilution solution as an osmotic buffer were studied.

\section{Materials and Methods}

\section{Source of embryos}

In vitro maturation, fertilization and culture procedures described by Van Soom et al. (1992) were slightly modified. Bovine ovaries were collected at an abattoir, and follicles $(2-8 \mathrm{~mm})$ were punctured and cumulus-oocyte complexes collected. Oocytes, surrounded by a complete cumulus oophorus, were matured in $500 \mu \mathrm{l}$ TCM199 bicarbonate buffered medium (Gibco, Merelbeke) supplemented with 20\% heat inactivated oestrous cow serum, $0.2 \mathrm{mmol}$ sodium pyruvate $\mathrm{l}^{-1}$ (Sigma, Bornem), $0.4 \mathrm{mmol}$ glutamine $\mathrm{l}^{-1}$ (Sigma), and $50 \mu \mathrm{g}$ gentamycin sulfate $\mathrm{ml}^{-1}$ (Gibco) for 24-26 h.

Fertilization was carried out using frozen-thawed semen that had been subjected to a discontinuous Percoll gradient to separate live spermatozoa. The in vitro fertilization medium consisted of $500 \mu \mathrm{l}$ Tyrode's solution (TALP) supplemented with $6 \mathrm{mg} \mathrm{BSA} \mathrm{ml}^{-1}, 20 \mu \mathrm{g}$ D-penicillamine, $10 \mu \mathrm{mol}$ hypotaurine $\mathrm{l}^{-1}, 1 \mu \mathrm{mol}$ adrenaline $\mathrm{ml}^{-1}$, and $25 \mu \mathrm{g}$ heparin $\mathrm{ml}^{-1}$, all obtained from Sigma. Sperm concentration was adjusted to $10^{6}$ spermatozoa $\mathrm{ml}^{-1}$. Incubation of spermatozoa with oocytes lasted for $20-24 \mathrm{~h}$. The maturation and fertilization media were not overlaid with oil.

Subsequently, the cumulus cells were mechanically removed, and the fertilized zygotes were transferred to $50 \mu$ ldroplets of oviduct coculture, overlaid with paraffin oil. The culture medium consisted of Ménézo-B2 (INRA, Paris) supplemented with $10 \%$ oestrous cow serum, $2.5 \mu \mathrm{g}$ fungizone $\mathrm{ml}^{-1}$ (Gibco) and a suspension of bovine oviduct epithelial cells (BOEC). Oviducts were collected from an abattoir and were dissected free from connective tissue. Oviductal epithelium was removed by scraping the lumen with a scalpel. Epithelial cells were washed three times with TALP medium and cultured overnight in TCM199 medium. Twenty-four hours later, the BOEC were washed again and $2 \mu \mathrm{l}$ of the cells ( \pm 20 oviduct vesicles) were added to each $50 \mu \mathrm{l}$ droplet of Ménézo-B2 medium. The droplets were prepared in a four-well multi-dish plate (Nunc, Roskilde). All cultures were performed at $39^{\circ} \mathrm{C}$, in $5 \% \mathrm{CO}_{2}$ in air with maximum humidity.

On day 6 after insemination, all embryos at the morula stage were transferred from the four wells of each multi-dish plate to one well. On the basis of the classification method of Mahmoudzadeh et al. (1993), grade 1 compact morula-early blastocysts, blastocysts and expanded blastocysts were selected for vitrification on day 7 after insemination. Embryos that did not develop to the morula stage on day 6, or were not selected as grade 1 embryos on day 7 but had developed to blastocyst or expanded blastocyst stages on day 8 are referred to as delayed embryos.

\section{Vitrification procedure}

The vitrification solution, EFS, described by Kasai et al. (1990) was used in the present experiments. The solution consists of $40 \%(\mathrm{v} / \mathrm{v})$ ethylene glycol (Sigma), 18\% (w/v) Ficoll (Ficoll 70, average $M_{\mathrm{r}}: 73$ 300; Pharmacia, Uppsala) and $10.26 \%(\mathrm{w} / \mathrm{v})$ sucrose dissolved in a holding medium (E. T. Freezing medium, Gibco) which is a modified phosphatebuffered solution supplemented with sodium pyruvate $\left(0.33 \mathrm{mmol} \mathrm{I}^{-1}\right)$, glucose $\left(5.6 \mathrm{mmol} \mathrm{l} \mathrm{l}^{-1}\right)$, BSA $(0.4 \%)$ and kanamycin sulfate $\left(0.025 \mathrm{mg} \mathrm{ml}^{-1}\right)$.

In the one-step method of vitrification, embryos from each group were transferred into Petri dishes (three to four embryos per dish), each containing $1.2 \mathrm{ml}$ EFS and then loaded into the straws. They were vitrified in liquid nitrogen after exposure to EFS for 1,3 or $5 \mathrm{~min}$. In the two-step vitrification method, the procedure described by Zhu et al. (1993) was followed with some modifications. Three to four embryos were placed into the first well of four-well multiplate dishes. This well contained $0.8 \mathrm{ml} 20 \%$ ethylene glycol (prepared in the holding medium) at $20 \pm 2{ }^{\circ} \mathrm{C}$. After exposure for $3 \mathrm{~min}$, the embryos were transferred to the second well, which contained $0.8 \mathrm{ml}$ EFS. They were then loaded into a straw and vitrified in liquid nitrogen within 30-45 s after exposure to EFS.

The $0.25 \mathrm{ml}$ French straws were loaded as follows: a $4 \mathrm{~cm}$ length was filled with $0.5 \mathrm{~mol}$ sucrose $1^{-1}$ (prepared in the holding medium) followed by a $1 \mathrm{~cm}$ air bubble, $0.5 \mathrm{~cm}$ vitrification solution, an air bubble (about $0.5 \mathrm{~cm}$ ), $2 \mathrm{~cm}$ EFS containing the embryos, an air bubble (about $0.5 \mathrm{~cm}$ ), $0.5 \mathrm{~cm}$ EFS, and an air bubble (about $1 \mathrm{~cm}$ ). The remaining part of the straw was filled with $0.5 \mathrm{~mol}$ sucrose $\mathrm{I}^{-1}$ and the straw was sealed with powder. For vitrification, the first part of the straw filled with sucrose $(4 \mathrm{~cm})$ was slowly immersed into liquid nitrogen; the remaining part of the straw was then plunged in.

\section{Recovery of embryos and assessment of their survival}

After a few days of storage in liquid nitrogen, the embryos were warmed to room temperature by placing the straw into a water bath at $20 \pm 2^{\circ} \mathrm{C}$ for $10 \mathrm{~s}$. Unless otherwise stated, warmed embryos were diluted in $0.25 \mathrm{~mol}$ sucrose $\mathrm{l}^{-1}$. The contents of each straw were emptied into a well of a sterile four-well multi-dish plate containing $0.7 \mathrm{ml} 0.25 \mathrm{~mol}$ sucrose $\mathrm{I}^{-\mathrm{I}}$. It took approximately $1 \mathrm{~min}$ to locate the embryos in the well and to transfer them to a second well, also containing $0.7 \mathrm{ml} 0.25 \mathrm{~mol}$ sucrose $\mathrm{l}^{-1}$. After $4 \mathrm{~min}$, the embryos were transferred into a well containing $0.7 \mathrm{ml}$ of the holding medium. Hereafter, embryos at each developmental stage of each group were cultured in a $50 \mu \mathrm{l}$ droplet of Ménézo-B2 medium supplemented with $10 \%$ oestrous cow serum and $2.5 \mu \mathrm{g}$ fungizone $\mathrm{ml}^{-1}$. With the exception of Expt 1 , the droplets were further supplemented with BOEC. In all experiments, dilution was performed at room temperature $\left(20 \pm 2^{\circ} \mathrm{C}\right)$. Survival after warming was defined as the percentage of 
Table 1. In vitro survival rate of in vitro produced embryos after three different exposure times to EFS with one-step vitrification

\begin{tabular}{|c|c|c|c|}
\hline \multirow[b]{2}{*}{ Embryonic stage } & \multicolumn{3}{|c|}{$\begin{array}{l}\text { Number of embryos } \\
\text { re-expanded/number vitrified (\%) }\end{array}$} \\
\hline & $1 \mathrm{~min}$ exposure & $3 \mathrm{~min}$ exposure & 5 min exposure \\
\hline Compact morula-early blastocyst & $28 / 52(54 \%)^{a}$ & $7 / 50(14 \%)^{b}$ & $4 / 47 \quad(9 \%)^{\mathrm{b}}$ \\
\hline Blastocyst & $26 / 50(52 \%)^{c}$ & $15 / 52(29 \%)^{\mathrm{d}}$ & $7 / 59(12 \%)^{d}$ \\
\hline Expanded blastocyst & $26 / 49(53 \%)^{\mathrm{e}}$ & $23 / 48(48 \%)^{\mathrm{e}}$ & $11 / 47(23 \%)^{f}$ \\
\hline
\end{tabular}

EFS: vitrification solution consisting of ethylene glycol, Ficoll and sucrose.

Values across rows with different superscripts are significantly different $(P<0.001)$.

${ }_{b}^{b}$, de In 3 min exposure column are significantly different $(P<0.01)$.

Data were pooled from seven replicates.

vitrified embryos that re-expanded or hatched during $72 \mathrm{~h}$ of culture.

\section{Experiment 1: effect of different exposure times to EFS}

The effect of exposure time of the embryos to the vitrification solution was studied by vitrifying embryos in liquid nitrogen after direct exposure to EFS for 1,3 or $5 \mathrm{~min}$. After recovery, they were cultured in Ménézo-B2 medium without BOEC.

\section{Experiment 2: importance of post-thaw culture media}

The beneficial effect of BOEC for culture of cryopreserved embryos in vitro, was demonstrated by vitrifying embryos at day 7 and 8 for 1 min after exposure to EFS. Warmed and diluted embryos were randomly divided into two groups. One group was cultured in Ménézo-B2 medium alone, the other in Ménézo-B2 medium supplemented with BOEC.

\section{Experiment 3: one-versus two-step vitrification}

The effect of stepwise addition of cryoprotectant was studied by randomly dividing the embryos into two groups. One group was vitrified following the one-step method and the other by the two-step method.

\section{Experiment 4: effect of sucrose dilution on vitrified embryos}

In this experiment, the effect of the sucrose concentration in the dilution medium was investigated. Embryos were vitrified by the two-step method. After warming, they were randomly diluted in three different sucrose solutions: $0,0.25$ and $0.5 \mathrm{~mol} \mathrm{l}^{-1}$.

\section{Statistical analyses}

The analysis of multiway tables of frequencies was performed according to a hierarchical loglinear procedure (Sokal and Rohlf, 1981) with the software program SPSS Release 5.0.
The significance of difference between two proportions was checked with a Chi-square test. A significance level of 0.05 was used unless stated otherwise.

\section{Results}

Results of embryo production in vitro

In a total of 31 replicates, 11228 oocytes were subjected to in vitro maturation and fertilization procedures. Presumed zygotes were cultured for 8 days. On day 3 , a total of 8310 $(74 \%)$ embryos had cleaved at least once and $4845(43 \%)$ had proceeded to the eight- to 16-cell stage. The total number of embryos that developed to the compact morulae to early blastocyst stages was $2759(25 \%)$. The number of day 7 grade I embryos selected from the 31 replicates was 1872 and the number of day 8 blastocysts and expanded blastocysts selected from ten replicates was 227 .

\section{Experiment 1: effect of different exposure times to EFS}

The survival rate of the morulae was significantly higher (54\%) after exposure to EFS for 1 min than that after 3 or $5 \mathrm{~min}$ (14\% and $9 \%$, respectively) $(P<0.0001)$ (Table 1$)$. There was no significant difference in the survival rate between 3 and 5 min of exposure. Blastocysts were sensitive to an increase of exposure time to EFS: $52 \%$ of blastocysts survived after $1 \mathrm{~min}$ exposure compared with $29 \%$ or $12 \%$ after 3 or $5 \mathrm{~min}$ exposure, respectively $(P<0.001)$. The effect on expanded blastocysts of prolonging exposure time was less pronounced compared with effects on morulae and blastocysts. There was no significant difference in the survival rate between exposure for $1 \mathrm{~min}(53 \%)$ and $3 \mathrm{~min}(48 \%)$. However, the survival rate dropped significantly when the exposure time was increased to $5 \mathrm{~min}(23 \%) \quad(P<0.001)$. No significant differences were detected between the three developmental stages after exposure for $1 \mathrm{~min}$ or $5 \mathrm{~min}$; however, there was a significant difference between the survival rate of the compact morulaeearly blastocysts, blastocysts and expanded blastocysts after exposure for $3 \mathrm{~min}(P<0.01)$. 
Table 2. In vitro survival rate of embryos at day 7 and day 8 after one-step vitrification and culture in Ménézo-B2 with or without BOEC

\begin{tabular}{|c|c|c|c|c|c|}
\hline \multirow[b]{2}{*}{ Age } & \multirow{2}{*}{$\begin{array}{l}\text { Embryos } \\
\text { Developmental stage }\end{array}$} & \multicolumn{2}{|c|}{$\begin{array}{l}\text { Number of embryos } \\
\text { re-expanded/number vitrified (\%) }\end{array}$} & \multicolumn{2}{|c|}{$\begin{array}{l}\text { Number of embryos } \\
\text { hatched/number vitrified (\%) }\end{array}$} \\
\hline & & $\mathrm{BOEC}(-)$ & $\mathrm{BOEC}(+)$ & $\operatorname{BOEC}(-)$ & $\operatorname{BOEC}(+)$ \\
\hline \multirow[t]{3}{*}{ Day 7} & Compact morula-early blastocyst & $39 / 77(51)$ & $47 / 73(64)$ & $3 / 77 \quad(4)$ & $9 / 73(12)^{\dagger}$ \\
\hline & Blastocyst & $45 / 87(52)^{a}$ & $60 / 82(73)^{b}$ & $11 / 87(13)^{c}$ & $25 / 82(30)^{\mathrm{d} t \dagger}$ \\
\hline & Expanded blastocyst & $45 / 78(58)$ & $43 / 67(64)$ & $6 / 78 \quad(8)^{c}$ & $22 / 67(33)^{\mathrm{ft \dagger}}$ \\
\hline \multirow[t]{2}{*}{ Day 8} & Blastocyst & $7 / 61(11)$ & $9 / 46(20)$ & $1 / 61$ & $2 / 46 \quad(4)$ \\
\hline & Expanded blastocyst & $5 / 63 \quad(8)$ & $7 / 57(12)$ & $0 / 63 \quad(0)$ & $1 / 57$ \\
\hline
\end{tabular}

BOEC: bovine oviduct epithelial cells.

Significant differences were as follows ${ }^{\text {ah }} P<0.01 ;{ }^{\text {cd }} P<0.01 ;{ }^{\text {e. }} \mathrm{P}<0.001$.

†Significantly different $(P<0.01)$ from ${ }^{\dagger \dagger}$.

Data were pooled from ten replicates.

Table 3. In vitro survival rate of embryos produced in vitro after one-step versus two-step vitrification and culture in Ménézo-B2 medium supplemented with BOEC

\begin{tabular}{lccccc}
\hline & \multicolumn{2}{c}{$\begin{array}{c}\text { Number of embryos } \\
\text { re-expanded/number vitrified (\%) }\end{array}$} & & \multicolumn{2}{c}{$\begin{array}{c}\text { Number of embryos } \\
\text { hatched/number vitrified (\%) }\end{array}$} \\
\cline { 2 - 3 } Embryonic stage & One-step & Two-step & & One-step & Two-step \\
\hline Compact morula-early blastocyst & $23 / 45(51)$ & $26 / 47(55)$ & & $5 / 45(11)$ & $8 / 47(17)$ \\
Blastocyst & $43 / 74(58)^{\mathrm{a}}$ & $59 / 79(75)^{\mathrm{b}}$ & & $14 / 74(19)^{\mathrm{e}}$ & $30 / 79(38)^{\mathrm{f}}$ \\
Expanded blastocyst & $30 / 59(51)^{\mathrm{c}}$ & $57 / 64(89)^{\mathrm{d}}$ & & $14 / 59(24)^{\mathrm{g}}$ & $44 / 64(69)^{\mathrm{h}}$ \\
\hline
\end{tabular}

BOEC: bovine oviduct epithelial cells.

Significant differences were as follows ${ }^{\mathrm{a}, \mathrm{b}} \mathrm{P}<0.05 ;{ }^{c, \mathrm{~d}} \mathrm{P}<0.001$; ${ }^{\mathrm{e}, \mathrm{f}} \mathrm{P}<0.01 ;{ }^{\mathrm{g} . \mathrm{h}} \mathrm{P}<0.001$

Data were pooled from eight replicates.

\section{Experiment 2: importance of post-thaw culture media}

The survival rate of blastocysts at day 7 significantly improved, from 52 to $73 \%(P<0.01)$, when the post-thaw culture medium was supplemented with BOEC (Table 2). A similar trend was noted with compact morulae-early blastocysts and expanded blastocysts at day 7 (from 51 to $64 \%$ and from 58 to $64 \%$, respectively), but the differences were not significant.

Supplementation of the post-thaw culture medium with BOEC had a positive effect on the hatching rate of the embryos. The increase in hatching rate was highly significant for expanded blastocysts at day 7 (from 8 to $33 \%, P<0.001$ ) and to a lesser extent for blastocysts (from 13 to $30 \%, P<0.01$ ) (Table 2). The hatching rate of compact morulae-early blastocysts at day 7 in the medium supplemented with BOEC was significantly lower $(12 \%)$ than that of the blastocysts and the expanded blastocysts (30 and $33 \%$, respectively) $(P<0.01)$.

Supplementing Ménézo-B2 with BOEC had no significant effect on the survival rate of embryos at day 8 . Blastocysts and expanded blastocysts had similar survival rates in both culture media (Table 2).

After vitrification, 'delayed embryos' showed a significantly lower survival rate than did normally developed embryos
$(P<0.001)$ in both media. The hatching rate of the delayed embryos' also significantly decreased $(P<0.05)$.

\section{Experiment 3: one-versus two-step vitrification}

The survival rate of embryos after two-step vitrification was significantly higher than after one-step vitrification. This was very pronounced for embryos at the expanded blastocyst stage $(P<0.001)$, but less pronounced for embryos at the blastocyst stage $(P<0.05)$. No significant difference was found between the survival rates of the compact morulae-early blastocysts following the two methods of vitrification (Table 3).

Two-step vitrification also had a positive effect on the rate of hatching of the surviving embryos, particularly at the expanded blastocyst $(P<0.001)$ and blastocyst stage $(P<0.01)$ (Table 3).

\section{Experiment 4: effect of sucrose concentration on vitrified embryos}

At any embryonic stage, the survival rate was independent of the sucrose concentration at the concentration used $(0,0.25$ and $0.5 \mathrm{~mol} \mathrm{l}^{-1}$ ) (Table 4). Irrespective of the sucrose concentration, the cumulative survival rate significantly decreased 
Table 4. In vitro survival rate of embryos produced in vitro after two-step vitrification, dilution in different sucrose solutions, and cultured in Ménézo-B2 medium supplemented with BOEC

Number of embryos re-expanded/number vitrified (\%) Number of embryos hatched/number vitrified (\%)

\begin{tabular}{|c|c|c|c|c|c|c|}
\hline \multirow[b]{2}{*}{ Embryonic stage } & \multicolumn{3}{|c|}{ Sucrose concentration $\left(\mathrm{mol} 1^{-1}\right)$} & \multicolumn{3}{|c|}{ Sucrose concentration $\left(\mathrm{mol} \mathrm{l}^{-1}\right)$} \\
\hline & 0 & 0.25 & 0.5 & 0 & 0.25 & 0.5 \\
\hline Compact morula-early blastocyst & $31 / 58(53)^{\mathrm{a}}$ & $32 / 61(52)^{a}$ & $33 / 57(58)^{a}$ & $12 / 58(21)^{d}$ & $14 / 61(23)^{d}$ & $12 / 57(21)^{\mathrm{d}}$ \\
\hline Blastocyst & $50 / 75(67)^{b}$ & $56 / 72(78)^{\mathrm{b}}$ & $48 / 71(68)^{b}$ & $30 / 75(40)^{\mathrm{e}}$ & $32 / 72(44)^{e}$ & $21 / 71(30)^{\mathrm{c}}$ \\
\hline Expanded blastocyst & $60 / 65(92)^{\mathrm{c}}$ & $63 / 67(94)^{c}$ & $50 / 60(83)^{c}$ & $48 / 65(74)^{f}$ & $46 / 67(69)^{f}$ & $41 / 60(68)^{f}$ \\
\hline
\end{tabular}

BOEC: bovine oviduct epithelial cells.

Values in rows with different superscripts are significantly different $\left(P<0.05\right.$ between values with superscripts ${ }^{d}$ and ${ }^{e} ; P<0.001$ for all others).

Data were pooled from six replicates.

when the embryos were vitrified at an earlier stage of development $(P<0.001)$.

\section{Discussion}

Previous experiments demonstrated that a vitrification solution composed of ethylene glycol, Ficoll and sucrose (EFS) for $3 \mathrm{~min}$ is sufficient for one-step vitrification of bovine compact morulae-early blastocysts produced in vivo, whereas in vitroderived embryos could not tolerate exposure for this time (Mahmoudzadeh et al, 1994a). However, this increased sensitivity of embryos produced in vitro was not only due to the vitrification procedure, but also to a greater extent to embryo quality. Changes were induced by the silicone oil, which was used to overlay the culture media (Mahmoudzadeh et al., unpublished; Van Soom et al., 1994), which made it very difficult to freeze or vitrify embryos generated. However, when the silicone oil used to overlay the culture medium was replaced by paraffin oil, the overall survival rate after one-step vitrification was improved from $5 \%$ to $30 \%$ in the present study.

Ethylene glycol is the only permeable component in the vitrification solution used in this report. Its low molecular mass permits a fast influx and efflux into and out of embryos (Szell et al., 1989; Voelkel and Hu, 1992a; Kuwayama et al., 1994). Thus, the exposure time of the embryo to the vitrification solution needs to be optimal to allow a sufficient amount of the cryoprotectant to permeate into the embryo and to avoid any toxic effects of longer exposure than necessary (Tachikawa et al., 1993).

In the present study, the survival rate of vitrified embryos decreased significantly from 53 to $30 \%$ when the exposure time to EFS was increased from 1 to $3 \mathrm{~min}$, and it further decreased to $14 \%$ when the exposure time was increased to $5 \mathrm{~min}$. These data indicate that prolonged exposure to ethylene glycol induces toxic effects in embryos produced in vitro. Our results also demonstrated that after exposure to EFS for $3 \mathrm{~min}$, embryos at a more advanced stage had a significantly higher survival rate. In many publications in which successful cryopreservation of embryos produced in vitro has been reported, embryos were often cryopreserved only at the blastocyst stage (Kajihara et al., 1992; Kuwayama et al., 1992, 1994; Massip et al., 1993; Tachikawa et al., 1993; Agca et al., 1994). However, in those reports in which the survival rate of embryos at different developmental stages after either conventional freezing (Zhang et al., 1993; Pollard and Leibo, 1994) or rapid cooling (Pollard and Leibo, 1994) or vitrification (Dinnyés et al., 1994) were compared, morulae produced in vitro were more sensitive to cryopreservation than were blastocysts or expanded blastocysts. The two most striking differences between the morula and the blastocyst stage are that the cells of the morula are slightly larger than the cells of the blastocyst, which might render them more sensitive to osmotic stress, induced by removal of the permeated cryoprotectant (Tachikawa et al., 1993), and that a large proportion of the water content of embryos at the morula stage is present inside the cells, whereas in blastocysts and expanded blastocysts the blastocoel contains the largest amount of the water. In the presence of a cryoprotectant solution, embryos with a blastocoel dehydrate faster owing to a lower membrane barrier against the water efflux. Sufficient dehydration of the morula can be achieved by increasing the exposure time. However, the embryo would be exposed to a higher extent of solution effect injury. The fundamental cellular differences between developmental stages of embryos produced in vitro (Pollard and Leibo, 1993) obviously result in a greater sensitivity of embryos to cryopreservation at earlier stages of development. Pollard and Leibo (1994) observed a slightly higher survival rate of cryopreserved morulae when the cooling rate was increased. They suggested that the extreme chilling injury of the embryos at earlier stages can be overcome by sufficiently rapid cooling. Our results, while supporting this suggestion, also demonstrate the importance of the solution effect injury.

Whereas Tachikawa et al. (1993) used a conditioned feeder layer of cumulus cells for embryo development after warming, we compared the effect of the presence or absence of feeder oviduct cells on embryo development after warming. Supplementing the culture medium with BOEC after warming was found to be beneficial for the survival rate of vitrified embryos produced in vitro. This effect was more pronounced for embryos at the blastocyst and expanded blastocyst stages, and confirms the results reported by Massip et al. (1993). It is possible that conditions for embryonic growth and development are optimal after transfer to a recipient shortly after warming. However, delayed embryos could not be rescued by 
improved culture conditions after warming. Embryos that are not recognized as compact morulae on day 6 , but still develop to blastocysts and expanded blastocysts after day 7, appear to be much more sensitive to the vitrification procedure than are embryos at the morula stage on day 6 that are vitrified on day 7. Similar results were obtained for blastocysts produced in vitro when they were either frozen (Del Campo et al., 1993; Han et al., 1994) or vitrified (Dinnyés et al., 1994; A. R. Mahmoudzadeh, A. Van Soom, P. Bols, M. T. Ysebaert and A. De Kruif, unpublished data) between day 6 and day 9 after insemination. Embryo quality, and in particular the number of blastomeres, was significantly lower in blastocysts that were not seen as a compact morula on day 6, than in normally developing embryos (Van Soom et al., 1993). This may explain their higher sensitivity to cryopreservation. There are no differences in morphology between normally developed blastocysts or expanded blastocysts and the delayed blastocysts.

Freezing and solution effect injuries induced by a conventional freezing method are probably more serious than those induced by vitrification (Fahy et al., 1984). However, during vitrification, embryos are exposed to a very concentrated solution of cryoprotectant that can produce irreversible damage in the cytoskeletal organization of the embryos (Overstrom et al., 1993). One possible method for reducing solution effect injury during vitrification is a stepwise exposure of embryos to a successively increasing concentration of cryoprotectant, combined with a subsequently decreasing exposure time. With this procedure, in vivo produced embryos of different species (Rall and Fahy, 1985; Massip et al., 1986; Smorag et al., 1989; Rall, 1992; Zhu et al., 1993) and also in vitro-derived bovine embryos (Kuwayama et al., 1992; Agca et al., 1994; Dinnyés et al., 1994) have been successfully cryopreserved. In the study reported here, survival rate of the embryos after vitrification was significantly increased from 54 to $75 \%$ by adding the vitrification solution in two steps. The survival rate of expanded blastocysts even reached $89 \%$ after two-step vitrification, with $69 \%$ hatching rate. Equally high survival rates were obtained by Zhu et al. (1993) for mouse expanded blastocysts produced in vivo and vitrified after exposure to 10 or $20 \%$ ethylene glycol for $5 \mathrm{~min}$, and then exposed to EFS for $30 \mathrm{~s}$.

Recent techniques for the dilution of thawed embryos, and in particular for dilution of vitrified-warmed embryos, use sucrose solution as an osmotic counterforce to restrict water permeation into the embryos and to prevent overswelling of the embryos while the cryoprotectant is leaving the cells. Sucrose concentration used is in the range from 0.25 to $1 \mathrm{~mol}$ $1^{-1}$ for 5-10 min. However, our results using solutions without sucrose, or with 0.25 or 0.5 mol sucrose $1^{-1}$, indicated that sucrose had no effect on embryo survival following two-step vitrification.

When ethylene glycol at a low concentration is used for conventional freezing of bovine embryos produced in vivo (Voelkel and Hu, 1992b), the high permeability of the cryoprotectant allows direct transfer of the thawed embryos without additional handling procedures. However, a high concentration of sucrose for dilution of vitrified embryos is recommended to prevent a prolonged exposure of the embryos to the vitrification solutions. The lowest concentration of sucrose used until now was reported by Dobrinsky et al. (1992).
They compared three different vitrification dilution treatments (0.1, 0.3 and $1 \mathrm{~mol} \mathrm{l}^{-1}$ ) for removal of glycerol and propylene glycol used for vitrification of embryos produced in vitro and found that dilution with $0.3 \mathrm{~mol}$ sucrose $1^{-1}$ was the optimal treatment.

To our knowledge, this is the first report demonstrating the possibility of diluting embryos produced in vitro and vitrified directly in holding medium without sucrose. This finding indicates that in straw dilution is possible; however, sufficient phosphate-buffered saline must be present in the straw to dilute the ethylene glycol to a less toxic concentration.

In conclusion, prolonged exposure of the embryos produced in vitro to vitrification solution had a detrimental effect on their survival. This effect was more pronounced in embryos undergoing vitrification at an earlier developmental stage. Supplementation of the post-thaw culture medium with BOEC significantly improved the survival rate of the vitrified embryos. Cryopreservation of delayed embryos was much less successful than that of normally developed embryos. Embryos at day 7 at the blastocyst and expanded blastocyst stage survived cryopreservation better, when EFS was added in two steps to the embryos as compared with one-step addition. Compact morulae-early blastocysts produced in vitro remained extremely sensitive to vitrification, and optimizing conditions, such as exposure time, post-thaw culture media and addition of cryoprotectant, did not improve their survival rates after warming. After two-step vitrification, embryos produced in vitro can be diluted directly into holding medium without sucrose mediation.

The Ministry of Culture and Higher Education of the Islamic Republic of Iran provided a grant (No. 44/28441) for A. R. Mahmoudzadeh. The authors thank A. Vanhoucke-De Medts and A. Van de Velde for excellent technical assistance and M. Coryn for critical reading of the manuscript.

\section{References}

Agca Y, Monson RL, Northey DL, Abas Mazni O and Rutledge JJ (1994) Post-thaw survival and pregnancy rates of in vitro produced bovine embryos after vitrification Theriogenology 41154 (Abstract)

Del Campo MR, Donoso MX, Palasz AT, Garcia A and Mapletoft RJ (1993) The effect of days in co-culture on survival of deep frozen bovine IVF blastocysts Theriogenology 39208 (Abstract)

Dinnyés A, Keefer CL, Stice SL, Solti L, Vajta G, Machaty Z and Rall WF (1994) Vitrification of IVMFC bovine embryos in VS3a and EFS solutions: a preliminary report Theriogenology 41189 (Abstract)

Dobrinsky JR, Stice SL, Phillips PE, Duby RT and Robl JM (1992) Development of IVM-IVF bovine embryos following vitrification dilution treatments Theriogenology 37202 (Abstract)

Fahy GM, MacFarlane DR, Angell CA and Meryman HT (1984) Vitrification as an approach to cryopreservation Cryobiology 21 407-426

Han YM, Yamashina H, Koyama N, Lee KK and Fukui Y (1994) Factors affecting viability of IVF-derived bovine blastocysts after freezing and thawing Theriogenology 41212 (Abstract)

Kajihara Y, Kometani N, Shitanaka Y, Saito S, Yamaguchi Y, Hishiyama K and Endo M (1992) Pregnancy rates and births after the direct transfer of frozenthawed bovine IVF embryos Theriogenology 37233 (Abstract)

Kasai M, Komi H, Takakamo A, Tsudera H, Sakurai T and Machida T (1990) A simple method for mouse embryo cryopreservation in a low toxicity vitrification solution, without appreciable loss of viability Journal of Reproduction and Fertility $\mathbf{8 9} 91-97$ 
Kasai M, Hamaguchi Y, Zhu SE, Miyake T, Sakurai T and Machida T (1992) High survival of rabbit morulae after vitrification in an ethylene glycol-based solution by a simple method Biology of Reproduction 46 1042-1046

Kuwayama M, Hamano S and Nagai T (1992) Vitrification of bovine blastocysts obtained by in vitro culture of oocytes matured and fertilized in vitro Journal of Reproduction and Fertility 96 187-193

Kuwayama M, Tasaka M and Hamano S (1994) In straw dilution of bovine IVF-blastocysts cryopreserved by vitrification Theriogenology 411231 (Abstract)

Leibo SP and Loskutoff NM (1993) Cryobiology of in vitro-derived bovine embryos Theriogenology 39 81-94

Mahmoudzadeh AR, Van Soom A, Van Vlaenderen I and de Kruif A (1993) A comparative study of the effect of one-step addition of different vitrification solutions on in vitro survival of vitrified bovine embryos Theriogenology 39 1291-1302

Mahmoudzadeh AR, Van Soom A and De Kruif A (1994a) Comparison of the survival rate of in vivo and in vitro produced bovine embryos after a simple vitrification procedure Vlaams Diergeneeskundig Tijdschrift 63 91-94

Mahmoudzadeh AR, Van Soom A, Ysebaert MT and De Kruif A (1994b) Comparison of two-step vitrification versus controlled freezing on survival of in vitro produced cattle embryos Theriogenology 42 1389-1397

Massip A, Van Der Zwalmen P, Scheffen B and Ectors F (1986) Pregnancies following transfer of cattle embryos preserved by vitrification Cryo-Letters 7 270-273

Massip A, Mermillod P, Wils C and Dessy F (1993) Effects of dilution procedure and culture conditions after thawing on survival of frozen bovine blastocysts produced in vitro Journal of Reproduction and Fertility 97 65-69

Overstrom EW, Duby RT, Dobrinsky JR, Robl JM, Baguisi A, Lonergan P, Duffy P, Walsh JH, Roche JF and Boland MP (1993) Cytoskeletal damage in vitrified or frozen bovine embryos Theriogenology 39276 (Abstract)

Pollard JW and Leibo SP (1993) Comparative cryobiology of in vitro and in vivo derived bovine embryos Theriogenology 39287 (Abstract)

Pollard JW and Leibo SP (1994) Chilling sensitivity of mammalian embryos Theriogenology 41 101 $\cdots 106$

Rall WF (1992) Cryopreservation of oocytes and embryos: methods and applications Animal Reproduction Science 28 237-245

Rall WF and Fahy GM (1985) Ice-free cryopreservation of mouse embryos at $-196^{\circ} \mathrm{C}$ by vitrification Nature $313573-575$
Shamsuddin M, Larsson B, Gustafsson H and Rodriguez-Martinez H (1994) A serum-free, cell-free culture system for development of bovine one-cell embryos up to blastocyst stage with improved viability Theriogenology 41 1033-1043

Smorag Z, Gajda B, Wieczorek B and Jura J (1989) Stage-dependent viability of vitrified rabbit embryos Theriogenology 31 1227-1231

Sokal RR and Rohlf FJ (1981) Biometry (2nd Edn) W. H. Freeman and Company, New York

Szell A, Shelton JN and Szell K (1989) Osmotic characteristics of sheep and cattle embryos Cryobiology 26 297-301

Tachikawa S, Otoi T, Kondo S, Machida T and Kasai M (1993) Successful vitrification of bovine blastocysts, derived by in vitro maturation and fertilization Molecular Reproduction and Development 34 266-271

Van Soom A, Van Vlaenderen I, Mahmoudzadeh AR, Deluyker $H$ and de Kruif A (1992) Compaction rate of in vitro fertilized bovine embryos related to the interval from insemination to first cleavage Theriogenology 38 905-919

Van Soom A, Mahmoudzadeh AR and De Kruif A (1993) Timing of bovine blastocyst formation and cell number of early blastocysts derived from in vitro technique Journal of Reproduction and Fertility Abstract Series 12117 (Abstract)

Van Soom A, Van Langendonckt A, Mahmoudzadeh AR, Deluyker H, Dessy F and De Kruif A (1994) Effect of oil quality on in vitro embryonic development in the bovine Theriogenology 41325 (Abstract)

Voelkel SA and Hu YX (1992a) Use of ethylene glycol as a cryoprotectant for bovine embryos allowing direct transfer of frozen-thawed embryos to recipient females Theriogenology 37 687-697

Voelkel SA and Hu YX (1992b) Direct transfer of frozen-thawed bovine embryos Theriogenology 37 23-37

Whittingham DG (1971) Survival of mouse embryos after freezing and thawing Nature 233 125-126

Wilmut I and Rowson LEA (1973) Experiments on the low-temperature preservation of cow embryos Veterinary Record 92 686-690

Zhang L, Barry DM, Denniston RS, Bunch TD and Godke RA (1993) Birth of live calves after transfer of frozen-thawed bovine embryos fertilised in vitro Veterinary Record 6 247-249

Zhu SE, Kasai M, Otoge H, Sakurai T and Machida T (1993) Cryopreservation of expanded mouse blastocysts by vitrification in ethylene glycol-based solutions Journal of Reproduction and Fertility 98 139-145 